\title{
Enumeration of Independent Sets in Benzenoid Chains
}

\author{
Mert Sinan $\mathrm{Oz}^{1}$, Ismail Naci Cangul ${ }^{2}$ \\ ${ }^{1}$ Faculty of Engineering and Natural Sciences, Department of Mathematics, \\ Bursa Technical University, 16320 Bursa, Turkey \\ sinan.oz@btu.edu.tr \\ ${ }^{2}$ Faculty of Arts and Science, Department of Mathematics, \\ Bursa Uludag University, 16059 Bursa, Turkey \\ cangul@uludag. edu.tr
}

(Received January 3, 2022)

\begin{abstract}
The Merrifield-Simmons index of a graph $G$ is defined as the summation of the number $i(G, k)$ of $k$-independent sets in $G$. It has applications in structural chemistry such as correlation with the thermodynamic properties of hydrocarbons. For this reason, enumeration of $i(G, k)$ of molecular graphs comes into prominence. In this paper, a method based on the transfer matrix technique is presented for enumerating $i(G, k)$ in benzenoid chains. As a consequence, for all $k \geq 0$, each $i(G, k)$ in arbitrary benzenoid chains is obtained via an appropriate product of three transfer matrices with dimension $5(k+1) \times 5(k+1)$ and a vector. In addition, we present two algorithms to make easier application of the method so that the applicability remains the same when the $k$ value increases.
\end{abstract}

\section{Introduction}

Let $G$ be a graph with vertex set $V$ and edge set $E$. A set consisting of all adjacent vertices to a vertex $v$ is called the neighborhood set of $v$, and it is denoted by $N_{G}(v)$. The union of the sets $N_{G}(v)$ and $\{v\}$ forms the closed neighborhood $N_{G}[v]$. If all vertices in a subset $S \subseteq V$ are pairwise nonadjacent, then $S$ is called an independent set. An independent set with $k$ vertices is called a $k$-independent set. The number of possible independent sets in $G$ with $k$ vertices is called the number of $k$-independent sets of $G$ and 
it is denoted by $i(G, k)$. In connection with $k$-independent sets, the Merrifield-Simmons index is defined as

$$
\sigma(G)=\sum_{k \geq 0} i(G, k)
$$

where $i(G, 0)=1$ and it is trivial that $i(G, 1)=|V|$.

The Merrifield-Simmons index has an important role in mathematical chemistry as it is used to predict thermodynamic properties of corresponding molecules by using molecular graphs, for a detailed survey, see $[7,8,16]$.

Hexagonal systems are molecular graphs of benzenoid hydrocarbons so that they are also called benzenoid systems. A benzenoid system is a 2-connected planar graph in which each finite region is a regular hexagon. In a benzenoid system, a vertex that is contained by three hexagons is called the internal vertex. A benzenoid system without any internal vertex is called catacondensed benzenoid system, [3]. If every hexagon is adjacent to at most two hexagons in a catacondensed benzenoid system, then it is called a benzenoid chain. Benzenoid chains are one of the prominent subclasses of catacondensed benzenoid systems and a great deal of mathematical and chemical studies are carried out on them. Some topological indices of benzenoid chains were studied in $[4,6,10,12,15]$. Extremal properties of hexagonal chains were studied in [9, 18-20].

The transfer matrix method has been used on several enumeration problems in combinatorial and chemical graph theory, $[13,14]$. Enumeration of independent sets in various molecular structures has been the topic of several studies. In $[1,2,17]$, the numbers of independent sets of small size in various fullerene graphs are computed. In [5], explicit formulae are presented for the number of independent sets of certain types of chain hexagonal cacti. Moreover, a method is presented for calculating the Merrifield-Simmons index of any benzenoid chains in [11]. However, for every $k \geq 0, i(G, k)$ values were not computed, separately. In this paper, we familiarize the $k$-independence vector at an edge of a hexagon of a given benzenoid chain and we use the transfer matrix technique to construct a method for enumerating $i(G, k)$ values in arbitrary benzenoid chains. The method is based on an appropriate multiplication of three transfer matrices with dimension $5(k+1) \times 5(k+1)$ and a vector with dimension $5(k+1) \times 1$, where $k \geq 0$. Furthermore, in Section 3, we design two algorithms to facilitate the application of the method as $k$ values increase.

Two significant recurrence relations for computing the number of $k$-independent sets 
in a graph $G$ are given as follows:

$$
i(S \cup H, k)=i(S, k) i(H, 0)+i(S, k-1) i(H, 1)+\cdots+i(S, 1) i(H, k-1)+i(S, 0) i(H, k),
$$

where $G=S \cup H$ and $S, H$ are two connected components of $G$. Moreover,

$$
i(G, k)=i(G-u z, k)-i\left(G-N_{G}[u]-N_{G}[z], k-2\right), e=u z \in G
$$
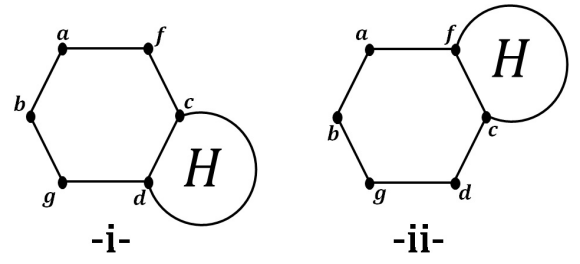

Figure 1. Benzenoid chain structures used in the next theorems

In Fig. 1, we show two isomorphic graphs with different vertex labels for the future use.

\section{Enumeration of $k$-independent sets in benzenoid chains}

Let us now introduce the $k$-independence vector of $G$ at a given edge to compute $i(G, k)$ of $G$.

Definition 2.1. Let $G=(V, E)$ be a graph and $e=u z \in E$. The $k$-independence vector of $G$ at $e=u z$ is as shown below:

$$
i_{u z}(G, k)=\left(\begin{array}{c}
i(G, k) \\
i(G, k-1) \\
\vdots \\
i(G, 0) \\
i\left(G-N_{G}[u], k\right) \\
i\left(G-N_{G}[u], k-1\right) \\
\vdots \\
i\left(G-N_{G}[u], 0\right) \\
i\left(G-N_{G}[[], k)\right. \\
i\left(G-N_{G}[z], k-1\right) \\
\vdots \\
i\left(G-N_{G}[z], 0\right) \\
i(G-u, k) \\
i(G-u, k-1) \\
\vdots \\
i(G-u, 0) \\
i(G-z, k) \\
i(G-z, k-1) \\
\vdots \\
i(G-z, 0)
\end{array}\right) .
$$


Theorem 2.1. Let $G=(V, E)$ be a graph obtained by fusing a graph $H$ and a hexagon with the edge cd in common (see Fig. 1 (i)). Then

$$
i_{a b}(G, k)=M_{1} \cdot i_{c d}(H, k)
$$

where $M_{1}$ is a transfer matrix with dimension $5(k+1) \times 5(k+1)$ as follows:

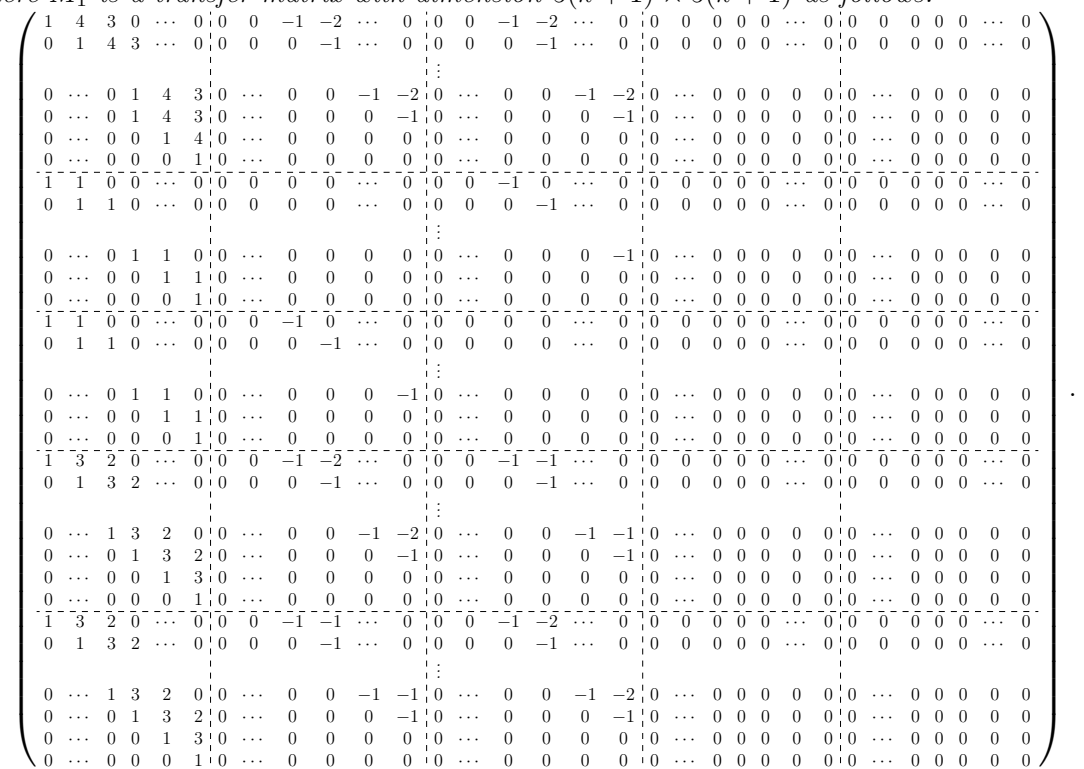

Proof. By the definition of the $k$-independence vector at the edge $a b$ of $G$, firstly let us aim to get the values $i(G, k), i\left(G-N_{G}[a], k\right), i\left(G-N_{G}[b], k\right), i(G-a, k)$ and $i(G-b, k)$ as a product of a vector and $i_{c d}(H, k)$. To achieve this purpose, let us delete suitable combinations of edges $c f$ and $d g$ from each corresponding subgraph. Thus, we can get desired equations by using the recurrence relations 1 and 2 as follows: 


$$
\begin{aligned}
& i(G, k)=i(G-c f-d g, k)-i\left(G-c f-N_{G}[d]-N_{G}[g], k-2\right)-i\left(G-N_{G}[c]-N_{G}[f], k-2\right) \\
& =i\left(P_{4} \cup H, k\right)-i\left(P_{2} \cup\left(H-N_{G}[d]\right), k-2\right)-i\left(P_{2} \cup\left(H-N_{G}[c]\right), k-2\right) \\
& =i(H, k)+4 i(H, k-1)+3 i(H, k-2)-i\left(H-N_{G}[d], k-2\right)-2 i\left(H-N_{G}[d], k-3\right) \\
& \text { - } \quad i\left(H-N_{G}[c], k-2\right)-2 i\left(H-N_{G}[c], k-3\right) \\
& =(1,4,3,0, \cdots, 0,0,0,-1,-2,0, \cdots, 0,0,0,-1,-2,0, \cdots, 0,0, \cdots, 0,0, \cdots, 0) \cdot i_{c d}(H, k), \\
& i\left(G-N_{G}[a], k\right)=i\left(G-N_{G}[a]-d g, k\right)-i\left(G-N_{G}[a]-N_{G}[d]-N_{G}[g], k-2\right) \\
& =i\left(P_{1} \cup H, k\right)-i\left(H-N_{G}[d], k-2\right) \\
& =i(H, k)+i(H, k-1)-i\left(H-N_{G}[d], k-2\right) \\
& =(1,1,0,0, \cdots, 0,0, \cdots, 0,0,0,-1,0, \cdots, 0,0, \cdots, 0,0, \cdots, 0) \cdot i_{c d}(H, k), \\
& i\left(G-N_{G}[b], k\right)=i\left(G-N_{G}[b]-c f, k\right)-i\left(G-N_{G}[b]-N_{G}[c]-N_{G}[f], k-2\right) \\
& =i\left(P_{1} \cup H, k\right)-i\left(H-N_{G}[c], k-2\right) \\
& =i(H, k)+i(H, k-1)-i\left(H-N_{G}[c], k-2\right) \\
& =(1,1,0,0, \cdots, 0,0,0,-1,0, \cdots, 0,0, \cdots, 0,0, \cdots, 0,0, \cdots, 0) \cdot i_{c d}(H, k), \\
& i(G-a, k)=i(G-a-c f-d g, k)-i\left(G-a-c f-N_{G}[d]-N_{G}[g], k-2\right) \\
& \text { - } i\left(G-a-N_{G}[c]-N_{G}[f], k-2\right) \\
& =i\left(P_{1} \cup P_{2} \cup H, k\right)-i\left(P_{1} \cup\left(H-N_{G}[d]\right), k-2\right)-i\left(P_{2} \cup\left(H-N_{G}[c]\right), k-2\right) \\
& =i(H, k)+3 i(H, k-1)+2 i(H, k-2)-i\left(H-N_{G}[d], k-2\right)-i\left(H-N_{G}[d], k-3\right) \\
& \text { - } \quad i\left(H-N_{G}[c], k-2\right)-2 i\left(H-N_{G}[c], k-3\right) \\
& =(1,3,2,0, \cdots, 0,0,0,-1,-2,0, \cdots, 0,0,0,-1,-1,0, \cdots, 0,0, \cdots, 0,0, \cdots, 0) \cdot i_{c d}(H, k), \\
& i(G-b, k)=i(G-b-c f-d g, k)-i\left(G-b-c f-N_{G}[d]-N_{G}[g], k-2\right) \\
& -\quad i\left(G-b-N_{G}[c]-N_{G}[f], k-2\right) \\
& =i\left(P_{1} \cup P_{2} \cup H, k\right)-i\left(P_{2} \cup\left(H-N_{G}[d]\right), k-2\right)-i\left(P_{1} \cup\left(H-N_{G}[c]\right), k-2\right) \\
& =i(H, k)+3 i(H, k-1)+2 i(H, k-2)-i\left(H-N_{G}[d], k-2\right)-2 i\left(H-N_{G}[d], k-3\right) \\
& \text { - } \quad i\left(H-N_{G}[c], k-2\right)-i\left(H-N_{G}[c], k-3\right) \\
& =(1,3,2,0, \cdots, 0,0,0,-1,-1,0, \cdots, 0,0,0,-1,-2,0, \cdots, 0,0, \cdots, 0,0, \cdots, 0) \cdot i_{c d}(H, k) .
\end{aligned}
$$

Then, by using the five obtained equations, it is deducable that the $k$ value decreases only for $i(G, k-1), \cdots, i(G, 0), i\left(G-N_{G}[a], k-1\right), \cdots, i\left(G-N_{G}[a], 0\right), i\left(G-N_{G}[b], k-\right.$ $1), \cdots, i\left(G-N_{G}[b], 0\right), i(G-a, k-1), \cdots, i(G-a, 0)$ and $i(G-b, k-1), \cdots, i(G-b, 0)$. Hence $i_{a b}(G, k)$ is obtained as the product of $i_{c d}(H, k)$ and a $5(k+1) \times 5(k+1)$ dimensional matrix whose the first, $(k+2)$-th, $(2 k+3)$-th,$(3 k+4)$-th and $(4 k+5)$-th rows are the vectors as follows, respectively:

$$
\begin{aligned}
& (1,4,3,0, \cdots, 0,0,0,-1,-2,0, \cdots, 0,0,0,-1,-2,0, \cdots, 0,0, \cdots, 0,0, \cdots, 0), \\
& (1,1,0,0, \cdots, 0,0, \cdots, 0,0,0,-1,0, \cdots, 0,0, \cdots, 0,0, \cdots, 0), \\
& (1,1,0,0, \cdots, 0,0,0,-1,0, \cdots, 0,0, \cdots, 0,0, \cdots, 0,0, \cdots, 0), \\
& (1,3,2,0, \cdots, 0,0,0,-1,-2,0, \cdots, 0,0,0,-1,-1,0, \cdots, 0,0, \cdots, 0,0, \cdots, 0),
\end{aligned}
$$


$(1,3,2,0, \cdots, 0,0,0,-1,-1,0, \cdots, 0,0,0,-1,-2,0, \cdots, 0,0, \cdots, 0,0, \cdots, 0)$.

The remaining rows of the matrix excluding the first, $(k+2)$-th, $(2 k+3)$-th, $(3 k+4)$ th and $(4 k+5)$-th rows, are determined in the echelon form in every 25 submatrices by depending on these rows since just $k$ value decreases in the equations of $i(G, k-1)$, $\cdots, i(G, 0), i\left(G-N_{G}[a], k-1\right), \cdots, i\left(G-N_{G}[a], 0\right), i\left(G-N_{G}[b], k-1\right), \cdots, i(G-$ $\left.N_{G}[b], 0\right), i(G-a, k-1), \cdots, i(G-a, 0)$ and $i(G-b, k-1), \cdots, i(G-b, 0)$. In conclusion, it is reached that $i_{a b}(G, k)=M_{1} \cdot i_{c d}(H, k)$ where $M_{1}$ is the given matrix above with dimension $5(k+1) \times 5(k+1)$.

As a natural result of Theorem 2.1, in the case of $S$ is isomorphic to path graph $P_{2}$, it is obtained that

$i_{a b}(G, k)=M_{1} \cdot\left[\begin{array}{lllllllllllllllllllllll}0 & \cdots & 0 & 2 & 1 & 0 & \cdots & 0 & 1 & 0 & \cdots & 0 & 1 & 0 & \cdots & 0 & 1 & 1 & 0 & \cdots & 0 & 1 & 1\end{array}\right]^{T}$.

Theorem 2.2. Let $G=(V, E)$ be a graph obtained by fusing a graph $H$ and a hexagon with the edge fc in common (see Fig. 1 (ii)). Then

$$
i_{a b}(G, k)=M_{2} \cdot i_{f c}(H, k)
$$

where $M_{2}$ is a transfer matrix with dimension $5(k+1) \times 5(k+1)$ as follows:

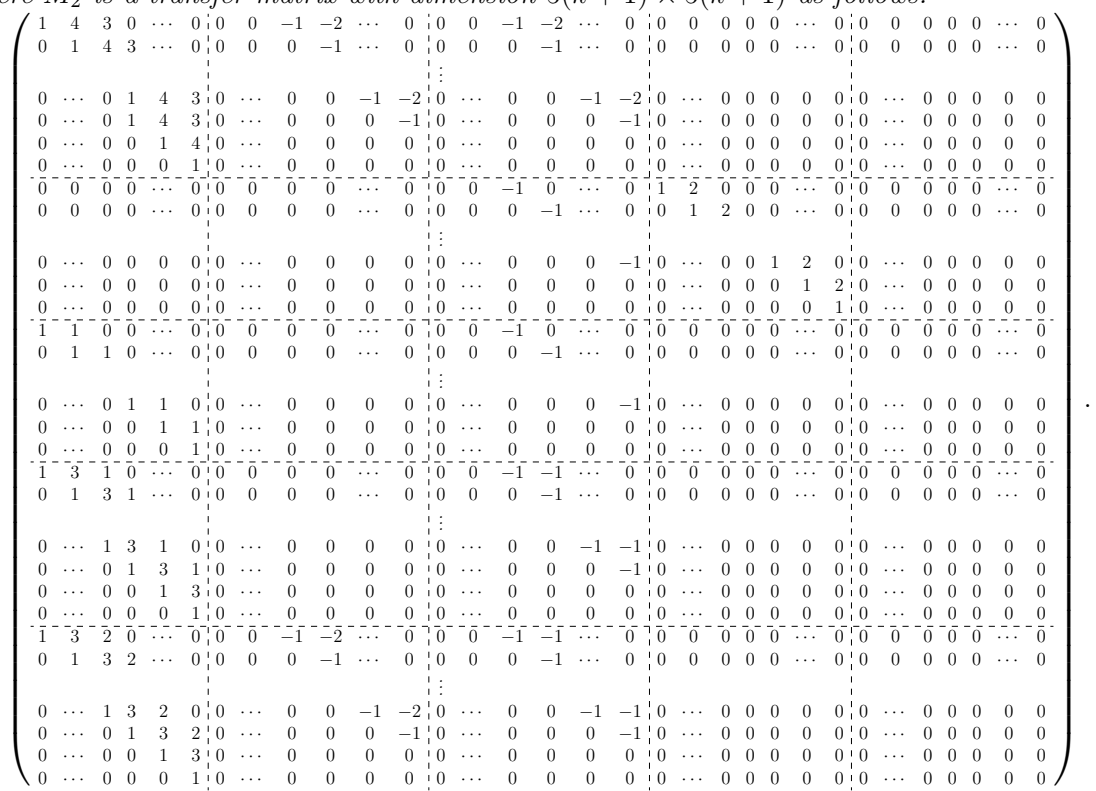


Proof. We need to compute $i(G, k), i\left(G-N_{G}[a], k\right), i\left(G-N_{G}[b], k\right), i(G-a, k)$ and $i(G-$ $b, k)$ by deleting appropriate combinations of edges $d c$ and $f a$ to get the subgraph $S$ apart from the hexagon. Afterwards, we utilize the recurrence relations 1 and 2 as the following:

$$
\begin{aligned}
& i(G, k)=i(G-f a-c d, k)-i\left(G-f a-N_{G}[c]-N_{G}[d], k-2\right)-i\left(G-N_{G}[f]-N_{G}[a], k-2\right) \\
& =i\left(P_{4} \cup H, k\right)-i\left(P_{2} \cup\left(H-N_{G}[c]\right), k-2\right)-i\left(P_{2} \cup\left(H-N_{G}[f]\right), k-2\right) \\
& =i(H, k)+4 i(H, k-1)+3 i(H, k-2)-i\left(H-N_{G}[c], k-2\right)-2 i\left(H-N_{G}[c], k-3\right) \\
& \text { - } i\left(H-N_{G}[f], k-2\right)-2 i\left(H-N_{G}[f], k-3\right) \\
& =(1,4,3,0, \cdots, 0,0,0,-1,-2,0, \cdots, 0,0,0,-1,-2,0, \cdots, 0,0, \cdots, 0,0, \cdots, 0) \cdot i_{f c}(H, k), \\
& i\left(G-N_{G}[a], k\right)=i\left(G-N_{G}[a]-c d, k\right)-i\left(G-N_{G}[a]-N_{G}[c]-N_{G}[d], k-2\right) \\
& =i\left(P_{2} \cup(H-f), k\right)-i\left(H-N_{G}[c], k-2\right) \\
& =i(H-f, k)+2 i(H-f, k-1)-i\left(H-N_{G}[c], k-2\right) \\
& =(0, \cdots, 0,0, \cdots, 0,0,0,-1,0, \cdots, 0,1,2,0, \cdots, 0,0, \cdots, 0) \cdot i_{f c}(H, k), \\
& i\left(G-N_{G}[b], k\right)=i\left(G-N_{G}[b]-c d, k\right)-i\left(G-N_{G}[b]-N_{G}[c]-N_{G}[d], k-2\right) \\
& =i\left(P_{1} \cup H, k\right)-i\left(H-N_{G}[c], k-2\right) \\
& \begin{array}{l}
=i(H, k)+i(H, k-1)-i\left(H-N_{G}[c], k-2\right) \\
=\quad(1,1,0,0, \cdots, 0,0, \cdots, 0,0,0,-1,0, \cdots, 0,0, \cdots, 0,0, \cdots, 0) \cdot i_{f c}(H, k),
\end{array} \\
& i(G-a, k)=i(G-a-c d, k)-i\left(G-a-N_{G}[c]-N_{G}[d], k-2\right) \\
& =i\left(P_{3} \cup H, k\right)-i\left(P_{1} \cup\left(H-N_{G}[c]\right), k-2\right) \\
& =i(H, k)+3 i(H, k-1)+i(H, k-2)-i\left(H-N_{G}[c], k-2\right)-i\left(H-N_{G}[c], k-3\right) \\
& =(1,3,1,0, \cdots, 0,0, \cdots, 0,0,0,-1,-1,0, \cdots, 0,0, \cdots, 0,0, \cdots, 0) \cdot i_{f c}(H, k), \\
& i(G-b, k)=i(G-b-f a-c d, k)-i\left(G-b-f a-N_{G}[c]-N_{G}[d], k-2\right)-i\left(G-b-N_{G}[f]-N_{G}[a], k-2\right) \\
& =i\left(P_{1} \cup P_{2} \cup H, k\right)-i\left(P_{1} \cup\left(H-N_{G}[c]\right), k-2\right)-i\left(P_{2} \cup\left(H-N_{G}[f]\right), k-2\right) \\
& =i(H, k)+3 i(H, k-1)+2 i(H, k-2)-i\left(H-N_{G}[c], k-2\right)-i\left(H-N_{G}[c], k-3\right) \\
& \text { - } \quad i\left(H-N_{G}[f], k-2\right)-2 i\left(H-N_{G}[f], k-3\right) \\
& =(1,3,2,0, \cdots, 0,0,0,-1,-2,0, \cdots, 0,0,0,-1,-1,0, \cdots, 0,0, \cdots, 0,0, \cdots, 0) \cdot i_{f c}(H, k) .
\end{aligned}
$$

From the five equations obtained above, other required equations in which only $k$ value decreases for $i(G, k-1), \cdots, i(G, 0), i\left(G-N_{G}[a], k-1\right), \cdots, i\left(G-N_{G}[a], 0\right), i\left(G-N_{G}[b], k-\right.$ $1), \cdots, i\left(G-N_{G}[b], 0\right), i(G-a, k-1), \cdots, i(G-a, 0)$ and $i(G-b, k-1), \cdots, i(G-b, 0)$ are obtained in the form of a product of a vector and $i_{f c}(H, k)$. Consequently, $i_{a b}(G, k)$ is reached as the product of $i_{f c}(H, k)$ and $5(k+1) \times 5(k+1)$ dimensional matrix whose the first, $(k+2)$-th, $(2 k+3)$-th, $(3 k+4)$-th and $(4 k+5)$-th rows are the vectors as given below, respectively:

$$
\begin{aligned}
& (1,4,3,0, \cdots, 0,0,0,-1,-2,0, \cdots, 0,0,0,-1,-2,0, \cdots, 0,0, \cdots, 0,0, \cdots, 0), \\
& (0, \cdots, 0,0, \cdots, 0,0,0,-1,0, \cdots, 0,1,2,0, \cdots, 0,0, \cdots, 0), \\
& (1,1,0,0, \cdots, 0,0, \cdots, 0,0,0,-1,0, \cdots, 0,0, \cdots, 0,0, \cdots, 0),
\end{aligned}
$$


$(1,3,1,0, \cdots, 0,0, \cdots, 0,0,0,-1,-1,0, \cdots, 0,0, \cdots, 0,0, \cdots, 0)$,

$(1,3,2,0, \cdots, 0,0,0,-1,-2,0, \cdots, 0,0,0,-1,-1,0, \cdots, 0,0, \cdots, 0,0, \cdots, 0)$.

Since only $k$ value decreases for other equations, the rest of the rows of the matrix exist in the echelon form in each of the 25 submatrices by depending on the stated rows above. Let us call the above matrix by $M_{2}$. Then we get the result as $i_{a b}(G, k)=M_{2} \cdot i_{f c}(H, k)$.

Theorem 2.3. Let $G=(V, E)$ be a graph obtained by fusing a graph $H$ and a hexagon with the edge cd in common (see Fig. 1). Then

$$
i_{b g}(G, k)=M_{3} \cdot i_{c d}(H, k)
$$

where $M_{3}$ is a transfer matrix with dimension $5(k+1) \times 5(k+1)$ as follows:

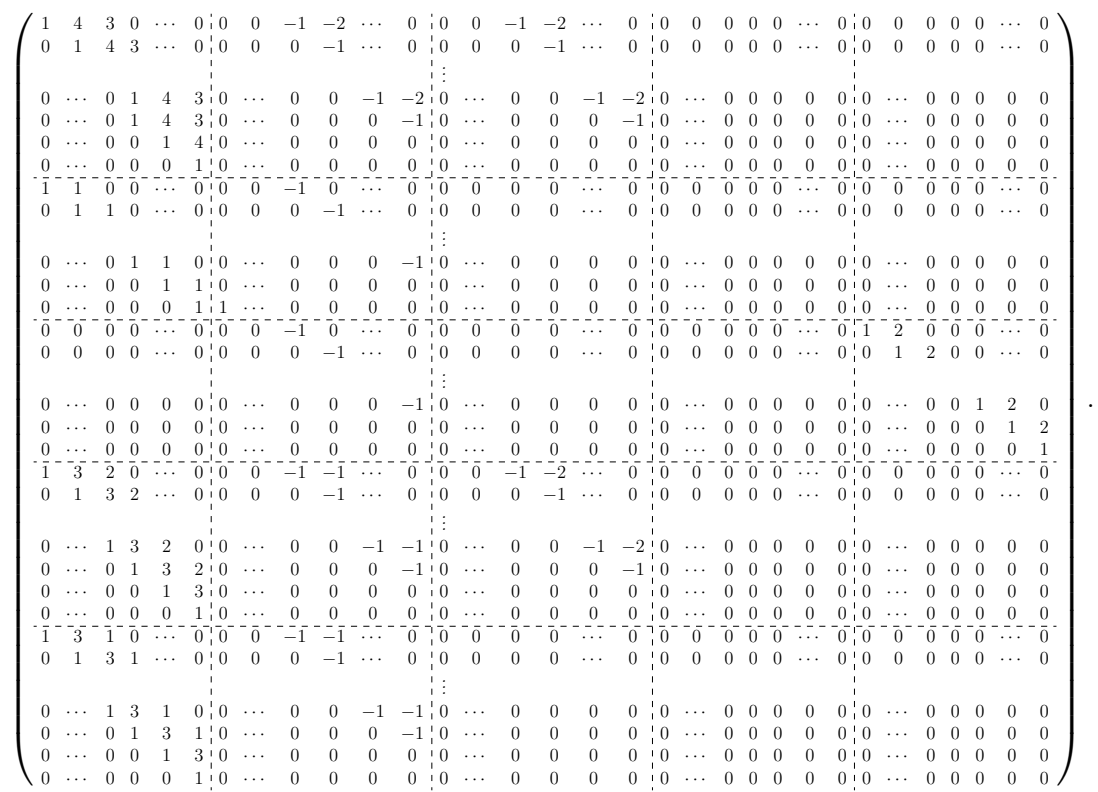

Proof. First of all, three of the values required for the proof have already been determined in the proof of Theorem 2.1 as presented below:

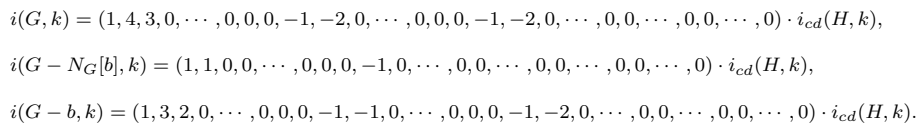


Hence, we need to compute just $i\left(G-N_{G}[g], k\right)$ and $i(G-g, k)$ by deleting the edge $c f$. Then by utilizing the recurrence relations 1 and 2 we get equations as below:

$$
\begin{aligned}
i\left(G-N_{G}[g], k\right) & =i\left(G-N_{G}[g]-c f, k\right)-i\left(G-N_{G}[g]-N_{G}[c]-N_{G}[f], k-2\right) \\
& =i\left(P_{2} \cup(H-d), k\right)-i\left(\left(H-N_{G}[c]\right), k-2\right) \\
& =i(H-d, k)+2 i(H-d, k-1)-i\left(H-N_{G}[c], k-2\right) \\
& =(0, \cdots, 0,0,0,-1,0, \cdots, 0,0, \cdots, 0,0, \cdots, 0,1,2,0, \cdots, 0) \cdot i_{c d}(H, k), \\
i(G-g, k) & =i(G-g-c f, k)-i\left(G-g-N_{G}[c]-N_{G}[f], k-2\right) \\
& =i\left(P_{3} \cup H, k\right)-i\left(P_{1} \cup\left(H-N_{G}[c]\right), k-2\right) \\
= & i(H, k)+3(H, k-1)+i(H, k-2)-i\left(H-N_{G}[c], k-2\right)-i\left(H-N_{G}[c], k-3\right) \\
= & (1,3,1,0, \cdots, 0,0,0,-1,-1,0, \cdots, 0,0, \cdots, 0,0, \cdots, 0,0, \cdots, 0) \cdot i_{c d}(H, k) .
\end{aligned}
$$

It is deducable that only the $k$ value decreases for the rest of the entries of the vector $i_{b g}(G, k)$, each of these values can be achieved as a product of $i_{c d}(H, k)$ and a $5(k+1) \times 1$ dimensional vector by using the equations above. Then it is clear that $i_{b g}(G, k)$ can be written as $M_{3} \cdot i_{c d}(H, k)$ where $M_{3}$ is a $5(k+1) \times 5(k+1)$ dimensional matrix whose the first, $(k+2)$-th, $(2 k+3)$-th, $(3 k+4)$-th and $(4 k+5)$-th rows are the following vectors, respectively, and remaining rows are in the echelon form in each of the 25 submatrices: $(1,4,3,0, \cdots, 0,0,0,-1,-2,0, \cdots, 0,0,0,-1,-2,0, \cdots, 0,0, \cdots, 0,0, \cdots, 0)$, $(1,1,0,0, \cdots, 0,0,0,-1,0, \cdots, 0,0, \cdots, 0,0, \cdots, 0,0, \cdots, 0)$, $(0, \cdots, 0,0,0,-1,0, \cdots, 0,0, \cdots, 0,0, \cdots, 0,1,2,0, \cdots, 0)$, $(1,3,2,0, \cdots, 0,0,0,-1,-1,0, \cdots, 0,0,0,-1,-2,0, \cdots, 0,0, \cdots, 0,0, \cdots, 0)$, $(1,3,1,0, \cdots, 0,0,0,-1,-1,0, \cdots, 0,0, \cdots, 0,0, \cdots, 0,0, \cdots, 0)$.

Consequently, this completes the proof.

\section{Algorithms}

In Section 2, we presented the theoretical part of our method used to compute the $k$ independent sets in benzenoid chains. This method is based on three transfer matrices and a vector that we called $M_{1}, M_{2}, M_{3}$ and $i_{c d}\left(P_{2}, k\right)$, respectively. However, when the $k$ value increases, computation is getting difficult because of the dimensions of the transfer matrices and the vector. Therefore, in this section we present two algorithms that are designed in MATLAB to easily get $M_{1}, M_{2}, M_{3}$ and $i_{c d}\left(P_{2}, k\right)$ for all $k \geq 0$. In the first algorithm, based on the input that is $k$ value, for a path graph $P_{2}$ with the edge $c d$, 
$i_{c d}\left(P_{2}, k\right)$ is obtained as follows:

$i_{c d}\left(P_{2}, k\right)=\left[\begin{array}{lllllllllllllllllllllll}0 & \cdots & 0 & 2 & 1 & 0 & \cdots & 0 & 1 & 0 & \cdots & 0 & 1 & 0 & \cdots & 0 & 1 & 1 & 0 & \cdots & 0 & 1 & 1\end{array}\right]^{T}$.

Let us present Algorithm 1 below:

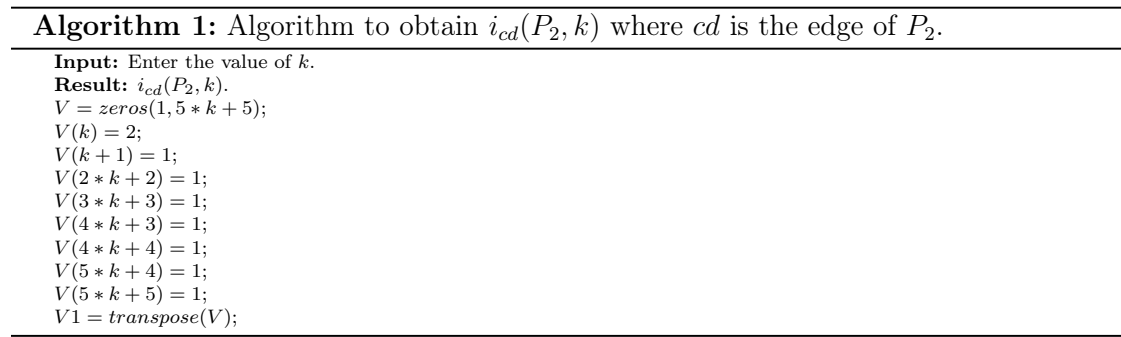

In the Algorithm 2, based on the input $k$ value, we get the transfer matrices $M_{1}, M_{2}, M_{3}$ by adding a couple of steps at the beginning before the step " $M(1,:)=T 1$; " as follows:

For the transfer matrix $M_{1}$, it is needed to add the steps consequtively as follows: $T 1=z \operatorname{eros}(1,5 * k+5) ; T 1(1)=1 ; T 1(2)=4 ; T 1(3)=3 ; T 1(k+4)=-1 ; T 1(k+5)=$ $-2 ; T 1(2 * k+5)=-1 ; T 1(2 * k+6)=-2 ; T 2=\operatorname{zeros}(1,5 * k+5) ; T 2(1)=$ $1 ; T 2(2)=1 ; T 2(2 * k+5)=-1 ; T 3=\operatorname{zeros}(1,5 * k+5) ; T 3(1)=1 ; T 3(2)=$ $1 ; T 3(k+4)=-1 ; T 4=z \operatorname{eros}(1,5 * k+5) ; T 4(1)=1 ; T 4(2)=3 ; T 4(3)=2 ; T 4(k+4)=$ $-1 ; T 4(k+5)=-2 ; T 4(2 * k+5)=-1 ; T 4(2 * k+6)=-1 ; T 5=\operatorname{zeros}(1,5 * k+$ 5); $T 5(1)=1 ; T 5(2)=3 ; T 5(3)=2 ; T 5(k+4)=-1 ; T 5(k+5)=-1 ; T 5(2 * k+5)=$ $-1 ; T 5(2 * k+6)=-2$;

For the transfer matrix $M_{2}$, it is needed to add the steps consequtively as follows: $T 1=\operatorname{zeros}(1,5 * k+5) ; T 1(1)=1 ; T 1(2)=4 ; T 1(3)=3 ; T 1(k+4)=-1 ; T 1(k+5)=$ $-2 ; T 1(2 * k+5)=-1 ; T 1(2 * k+6)=-2 ; T 2=z \operatorname{eros}(1,5 * k+5) ; T 2(2 * k+5)=$ $-1 ; T 2(3 * k+4)=1 ; T 2(3 * k+5)=2 ; T 3=z \operatorname{eros}(1,5 * k+5) ; T 3(1)=1 ; T 3(2)=$ $1 ; T 3(2 * k+5)=-1 ; T 4=z \operatorname{eros}(1,5 * k+5) ; T 4(1)=1 ; T 4(2)=3 ; T 4(3)=$ $1 ; T 4(2 * k+5)=-1 ; T 4(2 * k+6)=-1 ; T 5=z \operatorname{eros}(1,5 * k+5) ; T 5(1)=1 ; T 5(2)=$ $3 ; T 5(3)=2 ; T 5(k+4)=-1 ; T 5(k+5)=-2 ; T 5(2 * k+5)=-1 ; T 5(2 * k+6)=-1 ;$

For the transfer matrix $M_{3}$, it is needed to add the steps consequtively as follows: $T 1=\operatorname{zeros}(1,5 * k+5) ; T 1(1)=1 ; T 1(2)=4 ; T 1(3)=3 ; T 1(k+4)=-1 ; T 1(k+5)=$ $-2 ; T 1(2 * k+5)=-1 ; T 1(2 * k+6)=-2 ; T 2=\operatorname{zeros}(1,5 * k+5) ; T 2(1)=$ $1 ; T 2(2)=1 ; T 2(k+4)=-1 ; T 3=z \operatorname{eros}(1,5 * k+5) ; T 3(k+4)=-1 ; T 3(4 * k+5)=$ $1 ; T 3(4 * k+6)=2 ; T 4=z \operatorname{eros}(1,5 * k+5) ; T 4(1)=1 ; T 4(2)=3 ; T 4(3)=$ 
$2 ; T 4(k+4)=-1 ; T 4(k+5)=-1 ; T 4(2 * k+5)=-1 ; T 4(2 * k+6)=-2 ; T 5=$ $z \operatorname{zeros}(1,5 * k+5) ; T 5(1)=1 ; T 5(2)=3 ; T 5(3)=1 ; T 5(k+4)=-1 ; T 5(k+5)=-1 ;$

Now, let us present the Algorithm 2, which runs according to the inputs above:

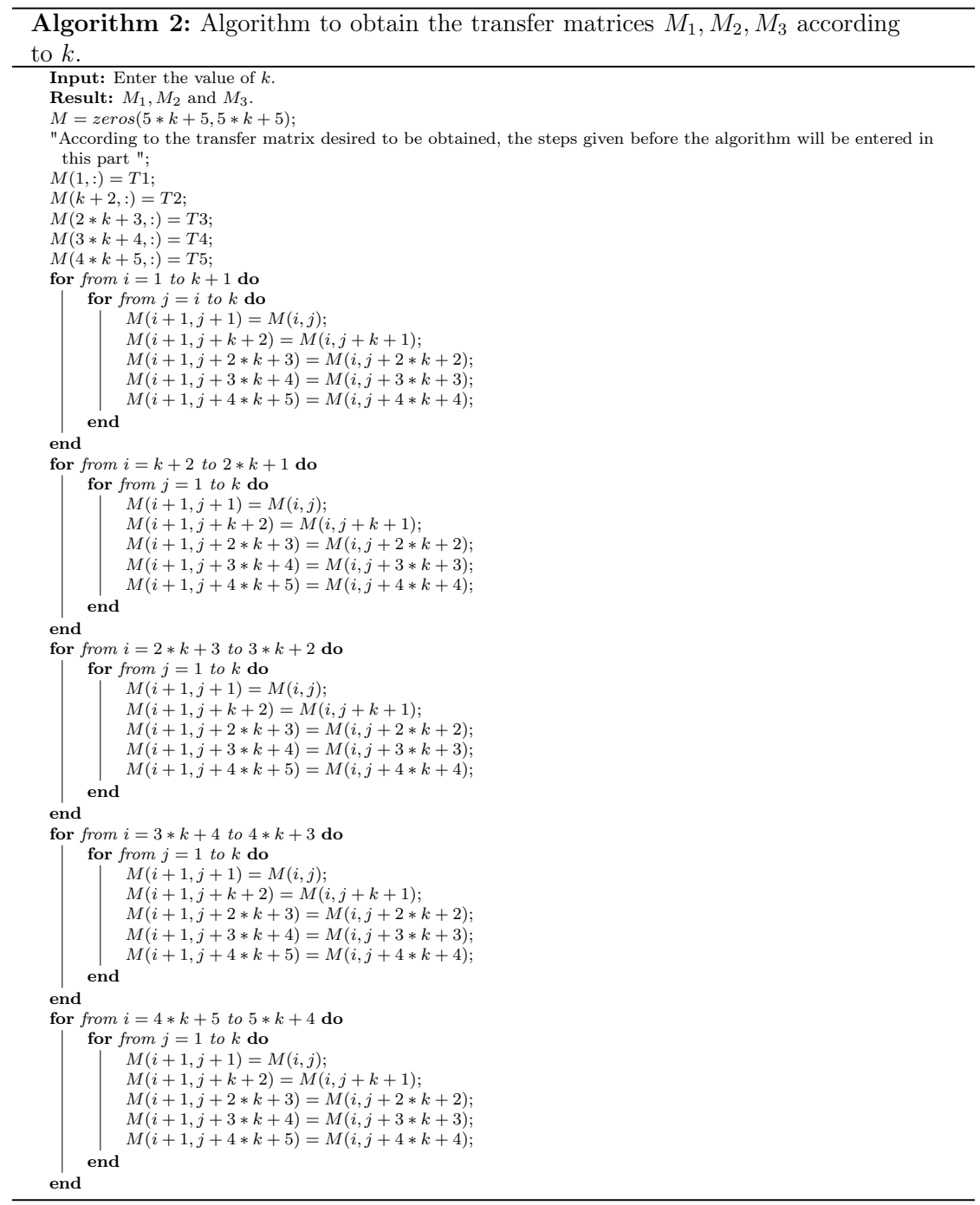

Example 3.1. Let $G$ be a benzenoid chain with 11 hexagons as shown in Fig. 2. 


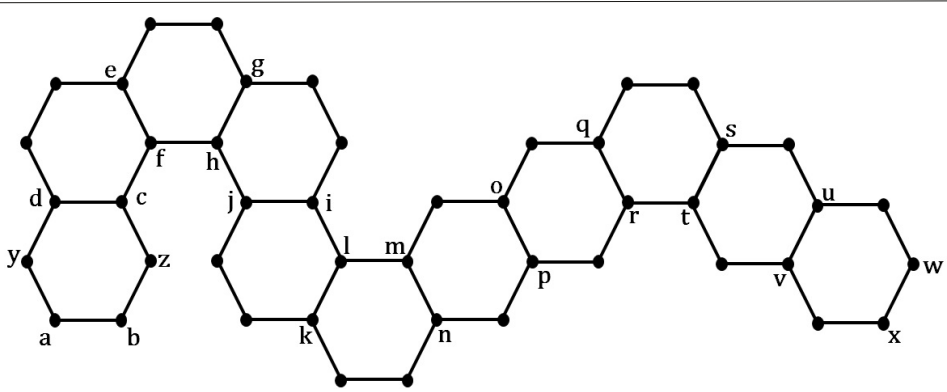

Figure 2. Benzenoid chain with 11 hexagons

For all $k \geq 0, i(G, k)$ of $G$ can be computed by means of the vector $i_{a b}(G, k)$ and Thms. 2.1, 2.2, 2.3 as follows:

$$
\begin{aligned}
i_{a b}(G, k) & =M_{1} \cdot i_{d c}\left(S^{I}, k\right), \\
& =M_{1} \cdot M_{3} \cdot i_{e f}\left(S^{I I}, k\right), \\
& =M_{1} \cdot M_{3} \cdot M_{3} \cdot i_{g h}\left(S^{I I I}, k\right), \\
& =M_{1} \cdot M_{3} \cdot M_{3} \cdot M_{3} \cdot i_{i j}\left(S^{I V}, k\right), \\
& =M_{1} \cdot M_{3} \cdot M_{3} \cdot M_{3} \cdot M_{2} \cdot i_{l k}\left(S^{V}, k\right), \\
& =M_{1} \cdot M_{3} \cdot M_{3} \cdot M_{3} \cdot M_{2} \cdot M_{2} \cdot i_{m n}\left(S^{V I}, k\right), \\
& =M_{1} \cdot M_{3} \cdot M_{3} \cdot M_{3} \cdot M_{2} \cdot M_{2} \cdot M_{1} \cdot i_{o p}\left(S^{V I I}, k\right), \\
& =M_{1} \cdot M_{3} \cdot M_{3} \cdot M_{3} \cdot M_{2} \cdot M_{2} \cdot M_{1} \cdot M_{1} \cdot i_{q r}\left(S^{V I I I}, k\right), \\
& =M_{1} \cdot M_{3} \cdot M_{3} \cdot M_{3} \cdot M_{2} \cdot M_{2} \cdot M_{1} \cdot M_{1} \cdot M_{3} \cdot i_{s t}\left(S^{I X}, k\right), \\
& =M_{1} \cdot M_{3} \cdot M_{3} \cdot M_{3} \cdot M_{2} \cdot M_{2} \cdot M_{1} \cdot M_{1} \cdot M_{3} \cdot M_{1} \cdot i_{u v}\left(S^{X}, k\right), \\
& =M_{1} \cdot M_{3} \cdot M_{3} \cdot M_{3} \cdot M_{2} \cdot M_{2} \cdot M_{1} \cdot M_{1} \cdot M_{3} \cdot M_{1} \cdot M_{1} \cdot i_{w x}\left(P_{2}, k\right),
\end{aligned}
$$

where $S^{I}, S^{I I}, \cdots, S^{X}$ are corresponding subgraphs.

Hence desired $k$ value can be chosen in the last equation above to get $i_{a b}(G, k)$. Since the vector $i_{a b}(G, k)$ contains the numbers of all $k$-independent sets in $G$ up to the $k$ value including $k$ value, all of these are obtained. Let us choose $k=25$ as follows:

$$
i_{a b}(G, 25)=M_{1} \cdot M_{3} \cdot M_{3} \cdot M_{3} \cdot M_{2} \cdot M_{2} \cdot M_{1} \cdot M_{1} \cdot M_{3} \cdot M_{1} \cdot M_{1} \cdot i_{w x}\left(P_{2}, 25\right),
$$


By using this equation together with Algorithms 1 and 2, the result is obtained as follows:

$\begin{array}{lllllll}i_{a b}(G, 25) & =[0 & 0 & 2 & 145 & 4268 & 68002 \\ 662662 & 4240055 & 18729664 & 59359916 & 139073582 & 246477981 & 336273579 \\ 357809386 & 299711119 & 198824106 & 104766545 & 43837794 & 14510552 & 3768649 \\ 757403 & 115209 & 12802 & 979 & 46 & 1 & 0 \\ 0 & 0 & 1 & 73 & 1960 & 29072 & 267747 \\ 1626798 & 6828646 & 20539053 & 45535285 & 76028515 & 97152079 & 96128659 \\ 74239291 & 44953503 & 21366091 & 7950163 & 2299484 & 510343 & 85075 \\ 10289 & 851 & 43 & 1 & 0 & 0 & 0 \\ 1 & 67 & 1981 & 30694 & 284616 & 1718068 & 7138367 \\ 21247946 & 46673132 & 77340774 & 98256520 & 96812984 & 74552134 & 45058462 \\ 21391547 & 7954496 & 2299974 & 510376 & 85076 & 10289 & 851 \\ 43 & 1 & 0 & 0 & 1 & 72 & 2308 \\ 38930 & 394915 & 2613257 & 11901018 & 38820863 & 93538297 & 170449466 \\ 239121500 & 261680727 & 225471828 & 153870603 & 83400454 & 35887631 & 12211068 \\ 3258306 & 672328 & 104920 & 11951 & 936 & 45 & 1 \\ 0 & 0 & 1 & 78 & 2287 & 37308 & 378046 \\ 2521987 & 11591297 & 38111970 & 92400450 & 169137207 & 238017059 & 260996402 \\ 225158985 & 153765644 & 83374998 & 35883298 & 12210578 & 3258273 & 672327 \\ 104920 & 11951 & 936 & 45 & 1]^{T} . & & \end{array}$

As a consequence, the entries starting from the first entry to the twenty-sixth entry give the following values, respectively:

$i(G, 25)=0, i(G, 24)=0, i(G, 23)=2, i(G, 22)=145, i(G, 21)=4268, i(G, 20)=$ 68002, $i(G, 19)=662662, i(G, 18)=4240055, i(G, 17)=18729664, i(G, 16)=59359916$, $i(G, 15)=139073582, i(G, 14)=246477981, i(G, 13)=336273579, i(G, 12)=357809386$, $i(G, 11)=299711119, i(G, 10)=198824106, i(G, 9)=104766545, i(G, 8)=43837794$, $i(G, 7)=14510552, i(G, 6)=3768649, i(G, 5)=757403, i(G, 4)=115209, i(G, 3)=$ $12802, i(G, 2)=979, i(G, 1)=46$ and $i(G, 0)=1$.

In addition, for $k=25$, the first and second entries of $i_{a b}(G, 25)$ are equal to zero and it means that the maximum $k$ value is 23 for nonzero $i(G, k)$. Therefore, by the definition of the Merrifield-Simmons index, the summation of $i(G, 23), i(G, 22), \cdots, i(G, 1)$ and $i(G, 0)$ gives the Merrifield-Simmons index of $G$. Hence, by summing the first, $\cdots$, twenty-sixth entries of $i_{a b}(G, 25)$, the Merrifield-Simmons index of $G$ is achieved as 1829004447. Note that, since $i(G, 24)=0$, the selection $k=23$ in $i_{a b}(G, k)$ will actually be sufficient for computing the Merrifield-Simmons index of $G$.

Alternatively, for all $k \geq 0$, the same $i(G, k)$ values can be obtained by using the following vectors and equations: 


$$
\begin{aligned}
& i_{y a}(G, 25)=M_{2} \cdot M_{3} \cdot M_{3} \cdot M_{3} \cdot M_{2} \cdot M_{2} \cdot M_{1} \cdot M_{1} \cdot M_{3} \cdot M_{1} \cdot M_{1} \cdot i_{w x}\left(P_{2}, 25\right), \\
& i_{b z}(G, 25)=M_{3} \cdot M_{3} \cdot M_{3} \cdot M_{3} \cdot M_{2} \cdot M_{2} \cdot M_{1} \cdot M_{1} \cdot M_{3} \cdot M_{1} \cdot M_{1} \cdot i_{w x}\left(P_{2}, 25\right) .
\end{aligned}
$$

The difference between the equations of the vectors $i_{a b}(G, 25), i_{y a}(G, 25)$ and $i_{b z}(G, 25)$ is just the $M_{1}, M_{2}, M_{3}$ matrices obtained due to the reduction difference in the first step.

Finally, $i(G, k)$ values can also be computed by using the $k$-independence vector of $G$ at the edge $x w$ as follows:

$$
i_{x w}(G, 25)=M_{1} \cdot M_{1} \cdot M_{2} \cdot M_{1} \cdot M_{1} \cdot M_{3} \cdot M_{3} \cdot M_{2} \cdot M_{2} \cdot M_{2} \cdot M_{1} \cdot i_{b a}\left(P_{2}, 25\right) .
$$

In conclusion, for all $k \geq 0, i(G, k)$ can be computed by using the $k$-independence vector and Thms. 2.1, 2.2, 2.3 for arbitrary benzenoid chains. Furthermore, for the maximum $k \geq 0$ such that $i(G, k) \neq 0$, since the sum of the rows of $i_{a b}(G, k)$ between the first and $(k+1)$-th corresponds to the Merrifield-Simmons index of $G$, this value is obtained by using the method. Similarly, the sum of the rows of $i_{a b}(G, k)$ between $k+2$ and $2 k+2,2 k+3$ and $3 k+3,3 k+4$ and $4 k+4,4 k+5$ and $5 k+5$ corresponds to the Merrifield-Simmons indices of $G-N_{G}[a], G-N_{G}[b], G-a, G-b$, respectively. Hence, all of these values are achieved by utilizing the method.

\section{References}

[1] A. Behmaram, H. Y. Azari, A. R. Ashrafi, On the number of paths, independent sets, and matchings of low order in (4,6)-fullerenes, MATCH Commun. Math. Comput. Chem. 69 (2013) 25-32.

[2] A. Behmaram, H. Y. Azari, A. R. Ashrafi, On the number of matchings and independent sets in (3,6)-fullerenes, MATCH Commun. Math. Comput. Chem. 70 (2013) 525-532.

[3] R. Cruz, C. A. Marín, J. Rada, Computing the Hosoya index of catacondensed hexagonal systems, MATCH Commun. Math. Comput. Chem. 77 (2017) 749-764.

[4] A. A. Dobrynin, Hexagonal chains with segments of equal lengths having distinct sizes and the same Wiener index, MATCH Commun. Math. Comput. Chem. 78 (2017) 121-132.

[5] T. Došlić, F. Måløy, Chain hexagonal cacti: Matchings and independent sets, Discr. Math. 310 (2010) 1676-1690.

[6] T. Došlić, A. Graovac, O. Ori, Eccentric connectivity index of hexagonal belts and chains, MATCH Commun. Math. Comput. Chem. 65 (2011) 745-752. 
[7] I. Gutman, O. E. Polansky, Mathematical Concepts in Organic Chemistry, Springer, Berlin, 1986.

[8] I. Gutman, S. J. Cyvin, Introduction to the Theory of Benzenoid Hydrocarbons, Springer, Berlin, 1989.

[9] I. Gutman, Extremal hexagonal chains, J. Math. Chem. 12 (1993) 197-210.

[10] N. Milosavljević, D. Stevanović, Detour index of hexagonal chains, MATCH Commun. Math. Comput. Chem. 72 (2014) 137-152.

[11] M. S. Oz, I. N. Cangul, Computing the Merrifield-Simmons indices of benzenoid chains and double benzenoid chains, J. Appl. Math. Comp., in press.

[12] M. S. Oz, I. N. Cangul, Computing the number of $k$-matchings in benzenoid chains, MATCH Commun. Math. Comput. Chem. 88 (2022) 79-92.

[13] O. E. Polansky, M. Randić, H. Hosoya, Transfer matrix approach to the Wiener numbers of cata-condensed benzenoids, MATCH Commun. Math. Comput. Chem. 24 (1989) 3-28.

[14] M. Randić, H. Hosoya, O. E. Polansky, On the construction of the matching polynomial for unbranched catacondensed benzenoids, J. Comput. Chem. 10 (1989) 683697.

[15] W. Shouzhong, B. Liu, A method of calculating the edge-Szeged index of hexagonal chain, MATCH Commun. Math. Comput. Chem. 68 (2012) 91-96.

[16] S. Wagner, I. Gutman, Maxima and minima of the Hosoya index and MerrifieldSimmons index: A survey of results and techniques, Acta Appl. Math. 112 (2010) $323-346$.

[17] Z. F. Wei, H. Zhang, Number of matchings of low order in (4,6)-fullerene graphs, MATCH Commun. Math. Comput. Chem. 77 (2017) 707-724.

[18] L. Z. Zhang, F. Zhang, Extremal hexagonal chains concerning $k$-matchings and $k$ independent sets, J. Math. Chem. 27 (2000) 319-329.

[19] L. Z. Zhang, F. Tian, Extremal catacondensed benzenoids, J. Math. Chem. 34 (2003) 111-122.

[20] Y. Zeng, F. Zhang, Extremal polyomino chains on k-matchings and k-independent sets, J. Math. Chem. 42 (2007) 125-140. 